\title{
Monochromatic light increases anthocyanin content during fruit development in bilberry
}

\author{
Laura Zoratti ${ }^{1}$, Marian Sarala ${ }^{1}$, Elisabete Carvalho ${ }^{2,3}$, Katja Karppinen ${ }^{1}$, Stefan Martens ${ }^{3}$, Lara Giongo ${ }^{3}$, \\ Hely Häggman ${ }^{1}$ and Laura Jaakola ${ }^{4,5^{*}}$
}

\begin{abstract}
Background: Light is one of the most significant environmental factors affecting to the accumulation of flavonoids in fruits. The composition of the light spectrum has been shown to affect the production of phenolic compounds during fruit ripening. However, specific information on the biosynthesis of flavonoids in fruits in response to different wavelengths of light is still scarce. In the present study bilberry (Vaccinium myrtillus L.) fruits, which are known to be rich with anthocyanin compounds, were illuminated with blue, red, far-red or white light during the berry ripening process. Following the illumination, the composition of anthocyanins and other phenolic compounds was analysed at the mature ripening stage of fruits.

Results: All the three monochromatic light treatments had significant positive effect on the accumulation of total anthocyanins in ripe fruits compared to treatment with white light or plants kept in darkness. The elevated levels of anthocyanins were mainly due to a significant increase in the accumulation of delphinidin glycosides. A total of 33 anthocyanin compounds were detected in ripe bilberry fruits, of which six are novel in bilberry (cyanidin acetyl-3-O-galactose, malvidin acetyl-3-O-galactose, malvidin coumaroyl-3-O-galactose, malvidin coumaroyl-3-Oglucose, delphinidin coumaroyl-3-O-galactose, delphinidin coumaroyl-3-O-glucose).
\end{abstract}

Conclusions: Our results indicate that the spectral composition of light during berry development has significant effect on the flavonoid composition of ripe bilberry fruits.

Keywords: Light quality, Vaccinium myrtillus L, Flavonoids, Anthocyanins, Bilberry, Berries, UPLC-MS/MS

\section{Background}

Anthocyanins, a class of flavonoid compounds, are the main pigments found in many flowers and fruits, in which they act as insect and animal attractants and protect the plant from light oxidative stress [1]. Furthermore, these metabolites are powerful antioxidants and therefore shown to be beneficial for human health [2]. Several reports have focused on their effects in the prevention of neuronal and cardiovascular diseases, cancer and diabetes as well as in promoting human nutrition $[2,3]$.

Bilberry (Vaccinium myrtillus L.) is among the most significant wild berry species in the Northern and Eastern Europe. Bilberry fruits are rich in phenolic acids, stilbenes and flavonoids, particularly in anthocyanins, which are

\footnotetext{
* Correspondence: laura.jaakola@uit.no

${ }^{4}$ Climate laboratory, Department of Arctic and Marine Biology, UiT the Arctic University of Norway, NO-9037 Tromsø, Norway

${ }^{5}$ Norwegian Institute for Agricultural and Environmental Research, Bioforsk Nord Holt, Box 2284, NO-9269 Tromsø, Norway

Full list of author information is available at the end of the article
}

estimated to represent nearly $90 \%$ of the total phenolics in these berries $[4,5]$. Anthocyanins are biosynthesized via the phenylpropanoid/flavonoid pathway consisting of a number of enzymatic steps that catalyze a sequential reaction leading to the production of different anthocyanidins including delphinidins $(\mathrm{Dp})$, cyanidins $(\mathrm{Cy})$, petunidins $(\mathrm{Pt})$, peonidins (Pn) and malvidins (Mv) (Additional file 1). In bilberry fruits, the quantitative and qualitative composition of flavonoids is known to be strongly affected by the fruit developmental stage [6,7]. Bilberry fruits are known to accumulate high yields of various anthocyanins both in skin and flesh during the ripening period, although genetic and environmental factors are also reported to affect the final composition [8-10]. Two families of transcription factors, the bHLH and MYB proteins, are strongly associated in the regulation of the anthocyanin pathway [11,12]. The phenylpropanoid pathway responds to various environmental stimuli such as temperature, photoperiod, soil fertility $[10,13,14]$ and light in particular $[15,16]$. 
Plants can sense multiple aspects of the light signals including light quantity (fluence), quality (wavelength), duration (photoperiod) and direction [17], which are perceived through at least four different families of photoreceptors, including phytochromes (red/far-red light receptors), cryptochromes and phototropins (blue light receptors) and UV-B photoreceptor (UVR8). These proteins perceive specific wavelengths of the visible light spectrum $(380-740 \mathrm{~nm})$ or the UV-light (280-315 nm) and transduce the signal to regulate photosynthesis, photomorphogenesis, phototropism, circadian rhythms as well as biosynthesis of secondary metabolites [18].

The induction of flavonoid and anthocyanin production by visible light has been extensively studied in several plant species, and it was found that the composition of light spectra regulated the biosynthesis of anthocyanins in Arabidopsis [19], cranberry (Vaccinium macrocarpon Ait.) [20], Gerbera [21], grape (Vitis vinifera L.) [22,23], lettuce (Lactuca sativa L.) [24], strawberry (Fragaria $x$ ananassa -Weston- Duchesne ex Rozier) [25] and turnip (Brassica napus L.) [26]. A significant increase in the amount of phenolic compounds has been seen in bilberry plants grown under direct sunlight when compared to plants grown under forest canopy $[9,15,27]$, but there is no information available on the effects of specific light wavelengths on their biosynthesis. Therefore, the aim of the present study was to analyze the influence of monochromatic wavelengths of the visible light spectrum on the production of phenolic compounds in bilberry fruit. Our particular interest was to study whether specific light wavelengths during berry development affect the biosynthesis and content of phenolic compounds. For this purpose, bilberry plants were illuminated with blue, red, far-red or white light during the berry ripening process and composition of the accumulated phenolic compounds was analyzed in ripe fruits. We also investigated the expression of key genes of bilberry flavonoid pathway in order to better understand the regulatory processes affecting biosynthesis of phenolic compounds during berry development.

\section{Results}

Characterization and quantification of phenolic compounds in ripe bilberry fruits

The phenolic compounds other than anthocyanins present in ripe bilberry fruits were analyzed by a UPLC-MS/MS method that has been earlier optimized for berry fruit species [28]. The phenolic compounds found in ripe bilberry fruits are listed in Table 1. The most abundant of those were hydroxycinnamic acids, namely chlorogenic acid and neochlorogenic acid. Naringenin (the precursor of flavonoid compounds) varied between 0.08 and $0.44 \mathrm{mg} / 100 \mathrm{~g}$ $\mathrm{DW}$, and was present in much higher concentration in the glycosylated form (naringenin 7-O-glucoside) which, to our knowledge, is reported for the first time in bilberry in the present study. Also among stilbenes, (-)-astringin was detected in this study for the first time to our knowledge in bilberry fruits. The flavone luteolin 7-O-glucoside was found only in trace amounts.

Ripe bilberries also contained flavonols, which included kaempferol 3-O-rutinoside, the quercetin derivatives (quercetin 3-O-glucose, quercetin 3-O-galactose, quercetin 3-O-glucuronide) and the myricetin derivatives (syringetin 3-O-glucose, syringetin 3-O-galactose and myricetin hexoses) in amounts comparable with earlier reports for bilberry [29].

The detected proanthocyanidins included monomers of catechin, epicatechin, epigallocatechin and gallocatechin. Among polymers, the most abundant was procyanidin B3 accompanied by lowers amounts of procyanidin A2, procyanidin B1, procyanidin B2 and/or B4 (which could not be separated using the present method [28]).

\section{Characterization and quantification of anthocyanins in ripe bilberry fruits}

Anthocyanins are the most abundant class of flavonoids present in ripe bilberry fruits. The anthocyanin content in ripe bilberry fruits was analyzed by a UPLC-MS/MS method which had been earlier optimized for grapevine [30]. The method was slightly modified to allow the detection of anthocyanidin galactosides and arabinosides that have earlier been described for bilberry (see Methods).

The total amount of anthocyanins in ripe berries varied between 1860 to $3397 \mathrm{mg} / 100 \mathrm{~g} \mathrm{DW}$, which is comparable with the amounts reported earlier for bilberry $[6,8]$. Altogether 33 anthocyanins were detected (Table 2), including the known 15 anthocyanins; Dp's, Cy's, Pt's, Pn's and Mv's combined with the sugars glucose, galactose and arabinose [8,31]. In addition, acetylated and p-coumaroyl-binded forms of anthocyanins, Pg's and Cy 3-O-sambubioside compounds were found. To our knowledge, some of the acetylated (Cy acetyl 3-O-galactose and $\mathrm{Mv}$ acetyl 3-O-galactose) and coumaroylated compounds (Dp coumaroyl 3-O-glucose, Dp coumaroyl 3-O-galactose, Mv coumaroyl 3-O-glucose, $\mathrm{Mv}$ coumaroyl 3-O-galactose) that were detected in this study have not been previously reported in bilberry fruits. Acetylated compounds were present in low amounts, with an average concentration between 0.05 to $0.72 \mathrm{mg} / 100 \mathrm{~g} \mathrm{DW}$ for the single compound detected (Table 2). The amount of $p$-coumaroylated anthocyanins was generally higher than the acetylated forms, even though the presence of these forms was more variable between the replicate plants. The contents ranged from the lowest of $\mathrm{Mv}$ coumaroyl 3-O-galactose to the highest of Pn and Mv coumaroyl 3-O-glucoside. However, the concentration of $\mathrm{Pn}$ and $\mathrm{Mv}$ coumaroyl 3-O-glucoside was in the same range with the known anthocyanins including $\mathrm{Pt}$ 3-O-glucoside, $\mathrm{Pt}$ 3-O-galactose, Mv 3- 
Table 1 Concentration of phenolic compounds ( $\mathrm{mg} / 100 \mathrm{~g} \mathrm{DW}$ ) detected in ripe bilberry fruits after monochromatic light treatment $(n=3)$

\begin{tabular}{|c|c|c|c|c|c|c|c|c|c|c|c|c|c|c|c|}
\hline \multirow[t]{2}{*}{ Compound } & \multicolumn{3}{|l|}{ Blue } & \multicolumn{3}{|l|}{ Red } & \multicolumn{3}{|c|}{ Far-red } & \multicolumn{3}{|c|}{ White } & \multicolumn{3}{|c|}{ Dark } \\
\hline & Av. & SD & St. & Av. & SD & St. & Av. & SD & St. & Av. & SD & St. & Av. & SD & St. \\
\hline Neochlorogenic acid & 80 & 35 & & 129 & 29 & & 105 & 27 & & 8 & 27 & & 96 & 21 & \\
\hline Chlorogenic acid & 113 & 59 & & 207 & 67 & & 162 & 58 & & 117 & 50 & & 135 & 36 & \\
\hline Total hydroxycinnamic acids & 193 & 94 & & 96 & 24 & & 168 & 55 & & 133 & 40 & & 100 & 24 & \\
\hline Naringenin & 0.3 & 0.2 & & 0.4 & 0.2 & & 0.2 & 0.1 & & 0.2 & 0.06 & & 0.2 & 0.1 & \\
\hline Naringenin 7 glucoside** & 82 & 26 & & 70 & 9 & & 69 & 7 & & 50 & 17 & & 68 & 29 & \\
\hline Total flavanones & 83 & 26 & & 70 & 9 & & 69 & 7 & & 50 & 17 & & 68 & 29 & \\
\hline$(-)$-Astringin** & 0.2 & 0.1 & & 0.2 & 0.1 & & 0.2 & 0.1 & & 0.1 & 0.06 & & 0.1 & 0.05 & \\
\hline Total stilbenes & 0.2 & 0.1 & & 0.2 & 0.1 & & 0.2 & 0.1 & & 0.1 & 0.06 & & 0.1 & 0.05 & \\
\hline Kaempferol 3 rutinoside & 4 & 1 & & 4 & 2 & & 5 & 1 & & 4 & 2 & & 4 & 3 & \\
\hline Quercetin $3 \mathrm{glu}$ & 5 & 3 & & 3 & 1 & & 4 & 2 & & 4 & 2 & & 4 & 2 & \\
\hline Quercetin 3 gal & 9 & 3 & $\mathrm{~b}$ & 17 & 2 & a & 15 & 10 & $a, b$ & 17 & 4 & a & 21 & 12 & $a, b$ \\
\hline Quercetin 3 glucuronide & 35 & 1 & & 46 & 9 & & 28 & 9 & & 42 & 5 & & 36 & 3 & \\
\hline Syringetin $3 \mathrm{gal}+\mathrm{glu}$ & 4 & 2 & & 6 & 6 & & 4 & 2 & & 4 & 0.5 & & 3 & 0.9 & \\
\hline Myricetin hexoses & 7 & 2 & $b$ & 12 & 1 & $a$ & 12 & 1 & a & 5 & 1 & $b$ & 8 & 3 & $b$ \\
\hline Total flavonols & 65 & 5 & & 86 & 19 & & 66 & 19 & & 76 & 10 & & 75 & 13 & \\
\hline Catechin & 1 & 0.8 & & 0.6 & 0.3 & & 2 & 1 & & 1 & 0.4 & & 0.7 & 0.3 & \\
\hline Epicatechin & 45 & 6 & & 45 & 19 & & 30 & 11 & & 43 & 7 & & 35 & 10 & \\
\hline Epigallocatechin & 11 & 1 & & 10 & 4 & & 9 & 5 & & 13 & 6 & & 11 & 3 & \\
\hline Gallocatechin & 15 & 1 & & 14 & 5 & & 12 & 6 & & 17 & 7 & & 14 & 4 & \\
\hline Procyanidin A2 & 0.25 & 0.07 & a & 0.08 & 0.06 & $b$ & 0.09 & 0.03 & $b$ & 0.25 & 0.10 & a & 0.23 & 0.18 & a \\
\hline Procyanidin B1 & 0.08 & 0.10 & $b$ & 0.15 & 0.12 & $b$ & 0.13 & 0.12 & $b$ & 0.24 & 0.02 & a & 0.11 & 0.08 & $b$ \\
\hline Procyanidin B2/B4 & 0 & 0 & & 1.32 & 1.32 & & 0.41 & 0.36 & & 0.23 & 0.23 & & 0.32 & 0.32 & \\
\hline Procyanidin B3 & 44 & 10 & & 45 & 16 & & 33 & 15 & & 41 & 6 & & 35 & 8 & \\
\hline Total proanthocyanidins & 116 & 16 & & 117 & 43 & & 86 & 37 & & 116 & 19 & & 96 & 21 & \\
\hline
\end{tabular}

glu = glucose, $\mathrm{gal}=$ galactose, $\mathrm{Av} .=$ average of three replicates, $\mathrm{SD}=$ standard deviation, $\mathrm{St} .=$ statistics .

The compounds marked with asterisk $\left(^{* *}\right)$ are first time detected in bilberry fruits to present. Significant differences by Tukey HSD (P < 0.05$)$ in response to the light treatments are marked by different letters for each compound and total amounts of compounds.

$\mathrm{O}$-glucoside, $\mathrm{Mv}$ 3-O-galactose, $\mathrm{Mv}$ 3-O-arabinose, $\mathrm{Pn}$ 3-O-glucoside, Pn 3-O-galactose and Pn 3-O-arabinose (Table 2). The amounts of $\mathrm{Pg}$ derivatives were low in bilberry fruits, $0.36 \mathrm{mg} / 100 \mathrm{~g}$ of $\mathrm{Pg} 3-\mathrm{O}$-glucoside and $0.11 \mathrm{mg} / 100 \mathrm{~g} \mathrm{DW}$ of Pg 3-O-galactose, while Pg 3-Oarabinose was not detected. The presence of Cy 3-O-sambubioside has also previously been reported in bilberry by $\mathrm{Du}$ et al. [32] in similar amounts that were found in our study.

Effect of monochromatic light on phenolic composition of ripe bilberry fruits

In order to investigate the effect of light quality on flavonoid accumulation in ripe berries, bilberry plants were treated with selected wavelengths of the visible light spectrum (blue, red, far-red or white light) during the fruit development process or left in the dark, as detailed in Figure 1. The effect of monochromatic light treatments during berry development on phenolic compounds in ripe berries is shown in Table 1. Significant variations $(\mathrm{P}<0.05)$ were detected in flavonols and proanthocyanidin compounds for some of the light treatments. The level of quercetin 3-O-galactose was significantly $(\mathrm{P}<0.05)$ lower in blue light treated plants compared with the other treatments. The levels of myricetin hexoses on the other hand were significantly higher under the red and far-red light treatments. On the contrary, the amounts of procyanidin A2 were lower under red and far-red light treatments, and procyanidin B1 level was higher under white light treatment compared with all the other light treatments.

\section{Monochromatic light affects anthocyanin composition of ripe bilberry fruits}

The most prominent effect of monochromatic light treatments was seen on anthocyanin content. Figure 2 shows 
Table 2 Concentration of anthocyanin compounds (mg/100 $\mathrm{g} \mathrm{DW}$ ) detected in ripe bilberry fruits after monochromatic light treatment $(n=3)$

\begin{tabular}{|c|c|c|c|c|c|c|c|c|c|c|c|c|c|c|c|}
\hline \multirow[t]{2}{*}{ Compound } & \multicolumn{3}{|l|}{ Blue } & \multicolumn{3}{|l|}{ Red } & \multicolumn{3}{|c|}{ Far-red } & \multicolumn{3}{|c|}{ White } & \multicolumn{3}{|l|}{ Dark } \\
\hline & Av. & SD & St. & Av. & SD & St. & Av. & SD & St. & Av. & SD & St. & Av. & SD & St. \\
\hline Cy acetyl 3 glu & 0.59 & 0.88 & & 0.49 & 0.64 & & 0.33 & 0.16 & & 0.54 & 0.38 & & 0.74 & 0.73 & \\
\hline Pt acetyl 3 glu & 0.10 & 0.09 & & 0.12 & 0.10 & & 0.08 & 0.01 & & 0.07 & 0.06 & & 0.26 & 0.45 & \\
\hline Pn acetyl 3 glu & 0.18 & 0.07 & & 0.11 & 0.09 & & 0.21 & 0.10 & & 0.39 & 0.24 & & 1.12 & 1.42 & \\
\hline Mv acetyl 3 glu & 0.35 & 0.30 & & 0.43 & 0.50 & & 1.04 & 0.53 & & 0.75 & 0.24 & & 4.54 & 4.54 & \\
\hline Cy acetyl 3 gal** & 0.10 & 0.18 & & 0.13 & 0.12 & & 0.17 & 0.16 & & 0.32 & 0.19 & & 0.59 & 0.56 & \\
\hline Mv acetyl 3 gal ${ }^{* *}$ & 0.17 & 0.11 & & 0.17 & 0.05 & & 0.34 & 0.16 & & 0.26 & 0.12 & & 0.52 & 0.52 & \\
\hline Dp acetyl 3 glu & 0.07 & 0.06 & & 0.05 & 0.09 & & 0.00 & 0.00 & & 0.03 & 0.05 & & 0.00 & 0.00 & \\
\hline Cy coum 3 glu & 10 & 3 & & 8 & 7 & & 11 & 4 & & 9 & 5 & & 24 & 27 & \\
\hline Dp coum 3 glu** & 1.8 & 0.3 & & 2.4 & 1.4 & & 1.6 & 1.6 & & 0.72 & 0.10 & & 2 & 2 & \\
\hline Pn coum 3 glu & 15 & 6 & & 18 & 15 & & 27 & 8 & & 28 & 13 & & 93 & 127 & \\
\hline Mv coum $3 \mathrm{glu}$ * $^{* *}$ & 24 & 9 & & 31 & 12 & & 41 & 5 & & 24 & 2 & & 121 & 181 & \\
\hline Cy coum 3 gal & 3 & 1 & & 4 & 0.6 & & 4.5 & 0.7 & & 5 & 2 & & 7 & 6 & \\
\hline Dp coum 3 gal** & 0.88 & 0.37 & & 0.87 & 0.34 & & 1.21 & 0.25 & & 0.64 & 0.15 & & 0.57 & 0.38 & \\
\hline Pn coum 3 gal & 0.69 & 0.28 & & 1.22 & 0.49 & & 1.63 & 0.20 & & 3.05 & 2.14 & & 3.32 & 3.32 & \\
\hline Mv coum 3 gal** & 0.22 & 0.21 & & 0.52 & 0.44 & & 0.97 & 0.22 & & 0.49 & 0.41 & & 1.96 & 1.96 & \\
\hline Cy sambubioside & 2.4 & 0.48 & & 2.3 & 1.9 & & 1.46 & 0.49 & & 1.91 & 1.21 & & 2.36 & 1.99 & \\
\hline Cy 3 glu & 600 & 174 & & 470 & 314 & & 557 & 71 & & 497 & 106 & & 554 & 103 & \\
\hline Dp 3 glu & 1135 & 29 & a & 1306 & 310 & a & 1075 & 96 & a & 665 & 164 & $b$ & 579 & 37 & $\mathrm{~b}$ \\
\hline Pt 3 glu & 24 & 5 & a & 28 & 1 & a & 25 & 6 & a & 15 & 2 & $b$ & 18 & 2 & $b$ \\
\hline PI 3 glu & 0.49 & 0.48 & & 0.16 & 0.28 & & 0.16 & 0.28 & & 0.49 & 0.49 & & 0.49 & 0.01 & \\
\hline Mv 3 glu & 47 & 1 & & 59 & 16 & & 69 & 21 & & 41 & 14 & & 57 & 16 & \\
\hline Pn 3 glu & 11 & 1 & & 10 & 3 & & 13 & 3 & & 12 & 2 & & 19 & 4 & \\
\hline Cy 3 gal & 214 & 44 & & 250 & 48 & & 248 & 44 & & 212 & 44 & & 197 & 17 & \\
\hline Dp 3 gal & 95 & 9 & $a$ & 100 & 5 & $a$ & 108 & 17 & a & 61 & 12 & $b$ & 47 & 3 & $b$ \\
\hline Pt 3 gal & 31 & 5 & $b$ & 42 & 4 & a & 43 & 5 & a & 25 & 6 & $b$ & 22 & 1 & $b$ \\
\hline Pl 3 gal & 0.05 & 0.04 & & 0.12 & 0.11 & & 0.09 & 0.08 & & 0.10 & 0.02 & & 0.19 & 0.08 & \\
\hline Mv 3 gal & 14 & 2 & $b$ & 31 & 10 & $\mathrm{a}$ & 33 & 16 & $a$ & 16 & 4 & $b$ & 20 & 5 & $a, b$ \\
\hline Pn 3 gal & 39 & 12 & & 55 & 36 & & 60 & 29 & & 53 & 17 & & 72 & 7 & \\
\hline Cy 3 ara & 159 & 42 & & 193 & 35 & & 152 & 39 & & 162 & 16 & & 145 & 27 & \\
\hline Dp 3 ara & 152 & 15 & $a$ & 167 & 16 & $a$ & 159 & 14 & $a$ & 90 & 18 & $b$ & 70 & 12 & $b$ \\
\hline Pt 3 ara & 43 & 7 & $a, b$ & 64 & 10 & a & 57 & 10 & a & 35 & 6 & $b$ & 32 & 11 & $b$ \\
\hline Mv 3 ara & 23 & 4 & $b$ & 48 & 15 & $\mathrm{a}$ & 46 & 17 & $\mathrm{a}$ & 25 & 2 & $b$ & 43 & 15 & $a$ \\
\hline Pn 3 ara & 11 & 3 & & 16 & 8 & & 18 & 12 & & 13 & 5 & & 17 & 8 & \\
\hline
\end{tabular}

glu = glucose, gal = galactose, ara = arabinose, coum = coumaroyl, Av. = average of three replicates, $\mathrm{SD}=$ standard deviation, $\mathrm{St}$. = statistics.

The compounds marked with asterisk $\left(^{* *}\right)$ are first time detected in bilberry fruits to present. Significant differences by Tukey HSD (P < 0.05$)$ in response to the light treatments are marked by different letters for each compound.

the effect of light treatments on the total amount of each class of anthocyanidins (Dp, Cy, Pn, Mv, Pt, Pg) calculated from the sum of the individual anthocyanin glycosides (Table 2). From the results it is evident that the content of Cy and Pn was not affected by the light treatments, whereas $\mathrm{Dp}, \mathrm{Mv}$ and $\mathrm{Pt}$ showed a significant $(\mathrm{P}<0.05)$ increase (33\%, $46 \%$ and $38 \%$, respectively) in berries of the plants treated with monochromatic light wavelengths when compared to the berries of the plants grown in white light conditions, suggesting that light quality affects the flavonoid pathway. The content of Mv showed a different behavior than Dp and Pt content; the concentration of $\mathrm{Mv}$ was significantly higher $(\mathrm{P}<0.05)$ in berries left in dark than under any of the light treatments (Figure 2). Table 2 shows effect of each of the light treatments on the accumulation of specific anthocyanin compounds. Red 

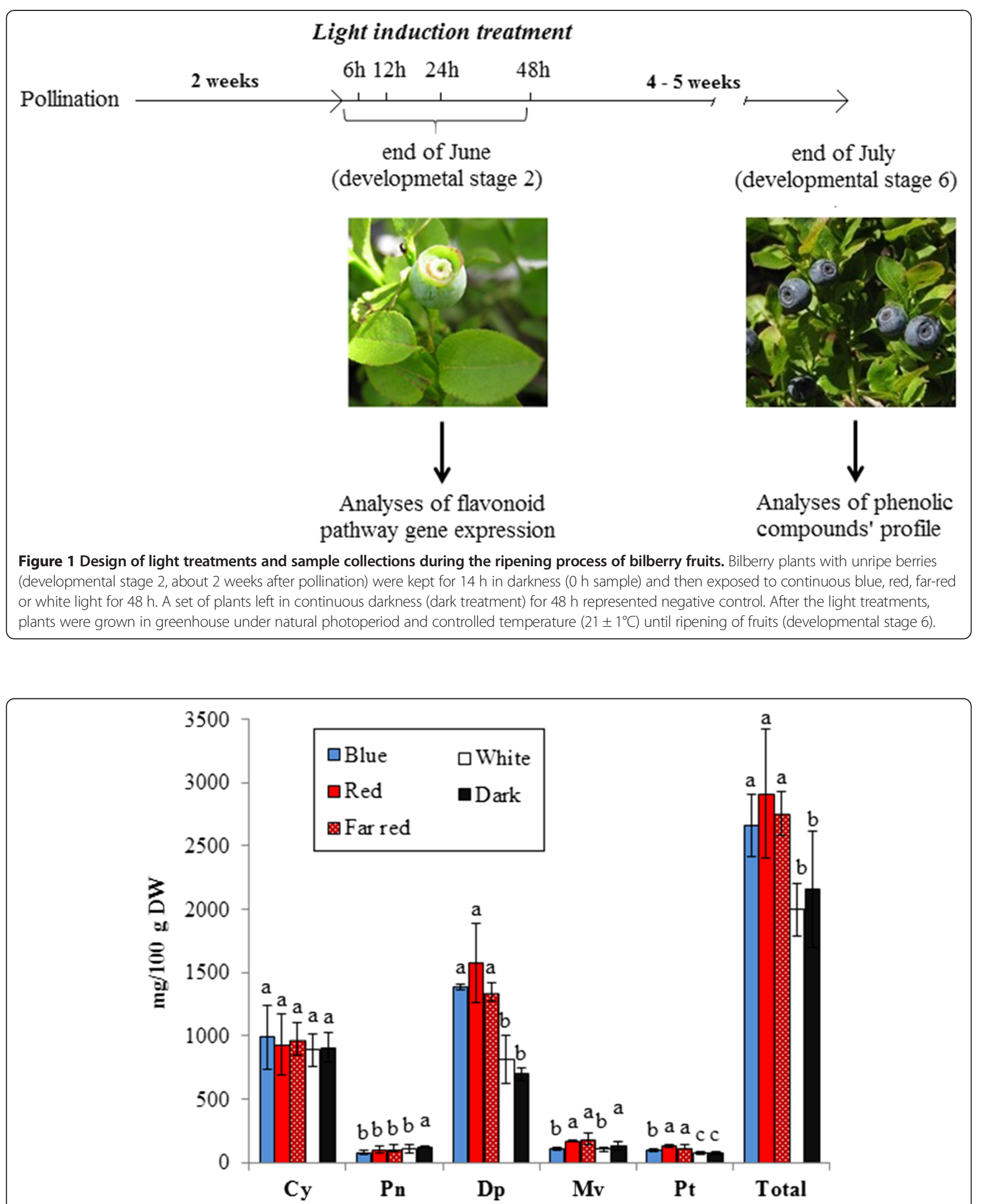

Figure 2 Concentration of anthocyanidin classes in ripe bilberry fruits treated with different light wavelengths (blue, red, far-red or white) or in dark conditions $(\mathbf{n}=\mathbf{3})$. Pg's are not reported here due to their low amounts compared to the other classes of anthocyanidins (Dp, Cy, Pt, Pn, Mv). For each class of anthocyanidin and the total amount of anthocyanins, significant differences by Tukey HSD $(P<0.05)$ in response to the light treatments are marked by different letters. 
and far-red light treatment increased $\mathrm{Dp}, \mathrm{Mv}$ and Pt compounds conjugated with glucose, galactose and arabinose sugars, but had no effect on the acylated and coumaroylated compounds. The same increase was induced by blue light, with the exception of $\mathrm{Pt} 3$-O-galactose, $\mathrm{Pt}$ 3-O-arabinose, $\mathrm{Mv}$ 3-O-galactose and $\mathrm{Mv}$ 3-O-arabinose.

The expression of flavonoid pathway genes $V m C H S$, $V m F 3^{\prime} 5^{\prime} H, V m D F R, V m A N S$ and VmANR, and the transcription factor $V m M Y B 2$ were also measured during the monochromatic light treatments at the stage of immature berries. The most of the examined genes showed increase in their expression during the first 12 hours of the study in the plants treated with monochromatic light compared with plants kept in darkness or under white light, even though variation between samples and time points was high (Additional file 2). However, VmANS showed the evident increase in the expression after 24 and 48 hours under monochromatic light when compared to dark treated plants. On the contrary, under white light, the expression was not increased compared to dark treated plants. Monochromatic light continued to up-regulate the expression of VmANS over dark treated plants throughout the light treatment until $48 \mathrm{~h}$, when the gene was increased up to 3-, 2- and 3.5-folds under blue, red and far-red light treatments, respectively, compared to dark treated plants. Under white light, the expression was only slightly increased (up to 1.3-fold) compared to dark treated plants (Additional file 2).

\section{Discussion}

Recent studies have shown that bilberry populations growing at northern latitudes contain higher amounts of flavonoids, in particular anthocyanins, in comparison to the southern populations $[8,9]$. The phenomenon is known to be under strong genetic control [9] even though environmental factors may also be involved in the regulation. Solar radiation is one of these factors, and it is known to increase the expression of the flavonoid biosynthesis genes and the content of flavonoids in bilberry leaves [15,31]. Moreover, higher amounts of anthocyanins were found in bilberry fruits grown in controlled conditions in a phytotrone in $24 \mathrm{~h}$ natural daylight, mimicking the light conditions of Arctic summers [10]. In the present study, the total anthocyanin content in ripe berries was significantly increased by monochromatic lights of blue, red and far-red, in comparison to fruits treated with white light or kept in darkness (Figure 2). Various effects of monochromatic light wavelengths on anthocyanin biosynthesis have also been reported in other species. For example, in turnip hypocotyls, far-red light had the most prominent effect on anthocyanin biosynthesis, comparable with the amount reached under sunlight [26]. In Gerbera, anthocyanin accumulation in flowers was particularly stimulated by blue light [21]. Blue light has been found to significantly increase the biosynthesis of anthocyanins also in fruit species, such as strawberries [25] and grape fruits $[22,23]$, while in cranberry fruits, red and far-red light increased the anthocyanin accumulation over white light [20].

A possible explanation of the present results can be found from the gene expression analyses of flavonoid pathway genes. The expression of the genes $V m C H S$, $V m F 3^{\prime} 5^{\prime} H, V m D F R$ and VmANR was less influenced by the light treatments (Additional file 2), which was consistent with the detected levels of flavanones, flavonols, stilbenes and proanthocyanidins in the berries kept under different light treatments (Table 1). Moreover, in earlier studies it has been shown that flavonoid pathway genes, for instance $C H S$, can have a diurnal rhythm $[33,34]$. This is one factor that can have affected the variation in the gene expression results between the different time points. On the contrary, the expression of $V m A N S$, which is the key gene in the biosynthesis of anthocyanins, shows a clear increasing trend under monochromatic light treatments, while white light and dark treatment does not have influence. Blue, red and far-red light all up-regulated the expression of VmANS already within the first $6 \mathrm{~h}$ after the beginning of the light treatment and also throughout the 2-day treatment (Additional file 2). According to Jaakola et al. [7], VmANS is expressed only at a very low level in bilberry fruits at the early stage of fruit development. However, the early stages of berry development appeared to be reactive to the light treatments in the present study. Monochromatic light treatments affected the accumulation of anthocyanins by increasing the expression of VmANS already at this early stage of berry development.

The higher amount of total anthocyanins in bilberry fruits in response to monochromatic light wavelengths was due to the increased production of Dp's and Pt's over Cy's and Pn's (Table 2, Figure 2). In the present study, the bilberry plants originated at the $65^{\circ} \mathrm{N}$ latitude and the amounts of Cy's and Dp's produced in plants treated with monochromatic lights were similar to the studies in which berries were grown in natural environment at similar latitudes $\left(64^{\circ} \mathrm{N}\right.$ [9] and $66^{\circ} \mathrm{N}$ [10]). Plants kept under white light or in darkness, showed a significant decrease in the content of Dp's, indicating that the spectral composition of light is involved in the accumulation of this class of anthocyanidins. Considering that in northern latitudes, summer nights are characterized by long twilight with high ratios of blue and far-red light [35], the present study emphasizes that northern light environment promote the accumulation of anthocyanins in bilberry already at the early stages of fruit ripening, by inducing qualitative and quantitative changes in anthocyanin content of ripe fruits. 


\section{Conclusions}

We showed that the treatment of bilberry plants under monochromatic light wavelengths of the visible light spectrum, for even short times during the ripening period of the fruits, is enough to induce a significant increase in the anthocyanin content in ripe fruits. Moreover, the quality of light affected particularly the biosynthesis of delphinidin glycosides. Our results indicate that the spectral composition of light regulates the accumulation of anthocyanins in fruits, showing an interaction between the flavonoid biosynthetic pathway and the composition of the light spectrum received by the plant.

\section{Methods}

\section{Plant material}

Bilberry (Vaccinium myrtillus L.) plants were harvested from three different locations I-III (I: $65^{\circ} 06^{\prime} \mathrm{N}, 25^{\circ} 5^{\prime} \mathrm{E}$; II: $65^{\circ} 04^{\prime} \mathrm{N}, 25^{\circ} 31^{\prime} \mathrm{E}$; III: $65^{\circ} 03^{\prime} \mathrm{N}, 25^{\circ} 28^{\prime} \mathrm{E}$ ) in forest stands in Finland. Plants were collected, in each location, within an area of $10 \mathrm{~m} \times 10 \mathrm{~m}$, assuming that the plants within this area belonged to the same genetic background [36] and thus represented specific ecotypes. Plants were collected at the stage when their fruits were small and green, presenting developmental stage 2 (Figure 1). Plants were harvested with their root system and were placed in boxes $(50 \mathrm{~cm} \times 70 \mathrm{~cm})$ containing forest peat soil.

After pollination, berries take usually six to seven weeks to ripe in natural stands of Finland. Bilberry fruit ripening stages were identified according to Jaakola et al. [6] and are presented in Figure 1. Developmental stage 2 represented small green unripe berries of 3 to $4 \mathrm{~mm}$ in size, approximately two weeks after pollination (end of June). At ripeness (developmental stage 6), which occurs about six weeks after pollination (end of July), the berries were 6 to $8 \mathrm{~mm}$ in diameter and turned to dark blue.

\section{Light sources}

Selador led lamps by PALETTA ${ }^{\mathrm{Tm}}$ (BMI supply, Queensbury, NY, USA) were used to irradiate plants with blue $(400-500 \mathrm{~nm})$, red $(600-700 \mathrm{~nm})$, far-red $(700-800 \mathrm{~nm})$ and white light (400-800 nm, Figure 3) wavelengths. The plants irradiated under blue light received a photon fluence rate of $8.10 \mu \mathrm{mol} \mathrm{m} \mathrm{m}^{-2} \mathrm{~s}^{-1}$, under red $7.8 \mu \mathrm{mol}$ $\mathrm{m}^{-2} \mathrm{~s}^{-1}$, under far-red $7.6 \mu \mathrm{mol} \mathrm{m}{ }^{-2} \mathrm{~s}^{-1}$ and under white

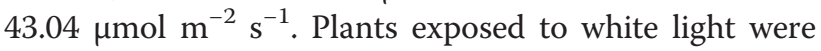
considered as a positive control. A set of plants kept in total darkness was considered as negative control. Light measurements were conducted by using USB RAD+ spectroradiometer (Ocean Optics Inc., Dunedin, FL, USA).

\section{Light treatments and sample collection}

Bilberry plants were treated with each specific light wavelength during the berry ripening period, as shown in Figure 1. Pools of bilberry plants from each location (I-III), were used for the treatments. Plants holding berries at stage 2, were initially kept in darkness for $14 \mathrm{~h}$ and then exposed to the continuous blue, red, far-red or white light induction or placed to darkness for $48 \mathrm{~h}$ (Figure 1). The berry developmental stage 2 was selected for the experiments based on preliminary analyses (data not shown) which indicated stage 2 to be the most reactive one, among all the bilberry fruit ripening stages, in the expression of flavonoid pathway genes in response to the light illumination. The light treatments were conducted

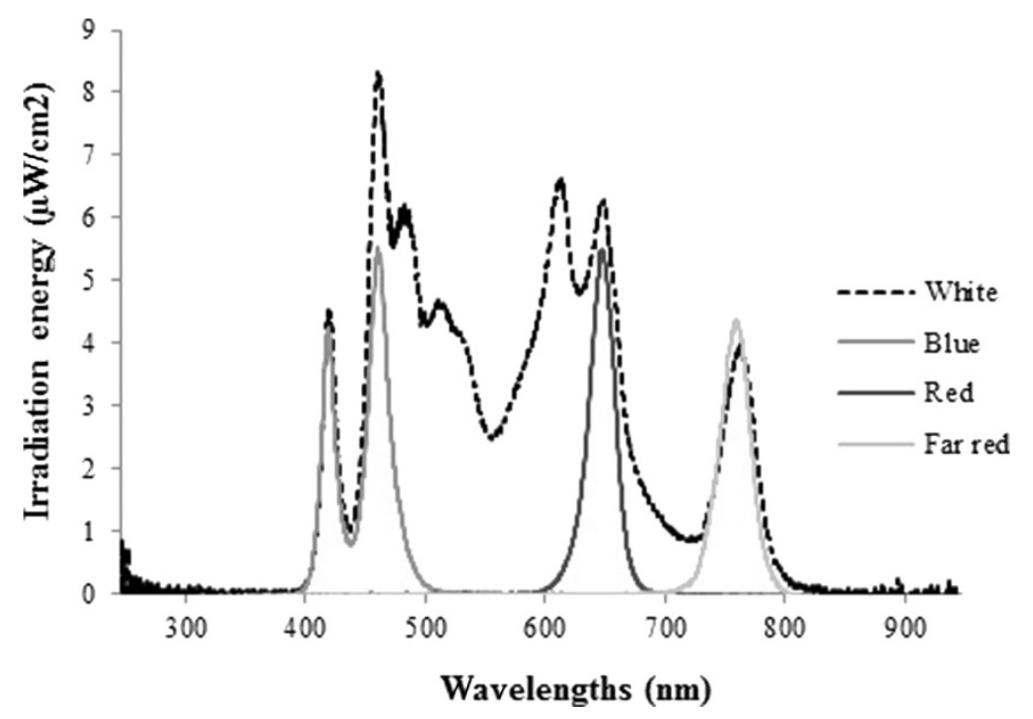

Figure 3 Light spectra used for the $\mathbf{4 8} \mathrm{h}$ light treatment experiments in bilberry plant. White, 400-800 nm; blue, 400-500 nm; red, 600-700 nm; and far-red, 700-800 nm. 
in growth chambers with controlled temperature $\left(21 \pm 1^{\circ} \mathrm{C}\right)$ and humidity (60\%) to erase the effect of temperature on flavonoid biosynthesis. After the light treatment, growth of plants was conducted in greenhouse under controlled temperature condition $\left(21 \pm 1^{\circ} \mathrm{C}\right)$ and natural photoperiod. When fully ripened (stage 6 , Figure 1 ), the berries were harvested and stored at $-80^{\circ} \mathrm{C}$ before freeze-dried within six months. The light treatments did not affect the process of ripening of the berries. Freezedried berries were stored in a desiccator at $-20^{\circ} \mathrm{C}$ until analysed for metabolic compounds.

\section{Metabolic analyses}

The ground material (100 mg out of $3 \mathrm{~g}$ ) of each sample was extracted with $1.5 \mathrm{~mL}$ of $80 \%$ methanol on shaking for $1 \mathrm{~h}$. Samples were centrifuged at $12000 \mathrm{~g}$ for $2 \mathrm{~min}$ (Sigma 3-30 k, Osterode, Germany) and the supernatants were collected. The extraction was repeated and the supernatants were combined and brought to a volume of $5 \mathrm{~mL}$. After filtering $(0.22 \mu \mathrm{m}$ PVDF filters $)$ and transferring to glass vials, the samples were randomized and analyzed for anthocyanins, flavonols, proanthocyanidins, stilbenes and other phenolic compounds by UPLCMS/MS.

\section{Analysis of phenolic compounds}

Flavonols, flavanones, hydroxycinnamic acids, proanthocyanidins and stilbenes were analysed as described in Vrhovsek et al. [28]. Chromatography, mass spectrometry conditions and multiple reaction monitoring (MRM) transitions can be found in the referred literature. Quantification was made by external calibration curves, injecting authentic standards of each of the detected compounds at different concentrations.

\section{Analysis of anthocyanins}

Anthocyanins were analysed by using UPLC-MS/MS as described by Arapitsas et al. [30]. Anthocyanins were detected by MRM, by screening the MS/MS transitions and using the parameters described in Additional file 3. For some of the compounds, there were no standards available, but they could be tentatively identified on the basis of their MRM transitions and the relative retention time, in respect to known compounds and considering previous results [37]. For example, standards of the galactoside derivatives of cyanidin and peonidin were available, and these compounds seem to elute before but closely to the respective glucoside derivatives (peaks 1, 2 and 22, 23 in Additional file 3). As such, the peak eluting 0.15 seconds before malvidin glucoside showing the same MRM transition is likely to be malvidin galactose (peak 15 in Additional file 3), and this reasoning can also be applied to the other galactoside and arabinoside derivatives.
For quantification, external calibration curves were prepared by injecting authentic standards of each compound at different concentrations. In case the authentic standard was not available, the anthocyanins were quantified relative to malvidin-3-O-glucose, using the malvidin-3-O-glucose calibration curve (Additional file 3).

\section{Statistical analysis}

The effect of the light treatment on every metabolite analyzed in the berries was tested with One-way ANOVA. Multiple comparisons were made by Tukey HSD's posthoc test. The tests were performed using STATISTICA version 12 .

\section{Supplementary analyses}

A supplementary study was conducted in order to study if the increased amount of anthocyanins was related to the gene expression of flavonoid pathway genes in bilberries (Additional file 1). Bilberry plants from locations I and II, with berries at developmental stage 2, were initially kept in darkness for $14 \mathrm{~h}$ ( $0 \mathrm{~h}$ sample) and then exposed to the continuous blue, red, far-red or white light induction or placed to darkness for $48 \mathrm{~h}$. During the light treatment, berry samples were collected for RNA isolation after $0,6,12,24$ and $48 \mathrm{~h}$ of treatment. Samples were immediately stored at $-80^{\circ} \mathrm{C}$ until analysed for gene expression.

\section{Isolation of RNA and CDNA preparation}

Total RNA was isolated from bilberry fruits at stage 2 that were collected after $0,6,12,24$ and $48 \mathrm{~h}$ from the beginning of the light treatments. The RNA was isolated according to the method of Jaakola et al. [38] with the exception that the phenol-chloroform extraction was substituted with the RNA purification protocol in E.Z. N.A. Total RNA Kit I (Omega Bio-Tek, Norcross, GA, USA). The quality of the isolated RNA was verified by measuring the absorbance spectrum with NanoDrop N-1000 spectrophotometer (NanoDrop Technologies, Thermo Scientific, Wilmington, DE, USA) and on a $1 \%$ $(\mathrm{w} / \mathrm{v})$ ethidium bromide-stained agarose gel. RNA was converted to cDNA with RevertAid Premium Reverse Transcriptase (Thermo Scientific) in accordance with the manufacturer's instruction. RNA extraction (and further gene expression analyses) was repeated twice for each set of plants.

\section{Gene expression analysis}

Transcript accumulation of the genes $\mathrm{VmCHS}, \mathrm{VmF3}$ '5' $^{\prime}$ $H, V m D F R, V m A N S$ and $V m A N R$, and the transcription factor $V m M Y B 2$ was detected using the LightCycler SYBR Green qPCR Kit (Roche Applied Sciences, Indianapolis, IN, USA). The primers used for the amplification are listed in Additional file 4. 
Analyses with qPCR were performed with a LightCycler 2.0 instrument and software (Roche). The PCR conditions were $95^{\circ} \mathrm{C}$ for $10 \mathrm{~min}$, followed by 45 cycles of $95^{\circ} \mathrm{C}$ for $10 \mathrm{~s}, 60^{\circ} \mathrm{C}$ for $20 \mathrm{~s}$, and $72^{\circ} \mathrm{C}$ for $10 \mathrm{~s}$. VmACT gene (Additional file 4) was used as a reference gene for relative quantification. Differential gene expression levels were calculated by comparing each of treatments to treatment $0 \mathrm{~h}$.

\section{Additional files}

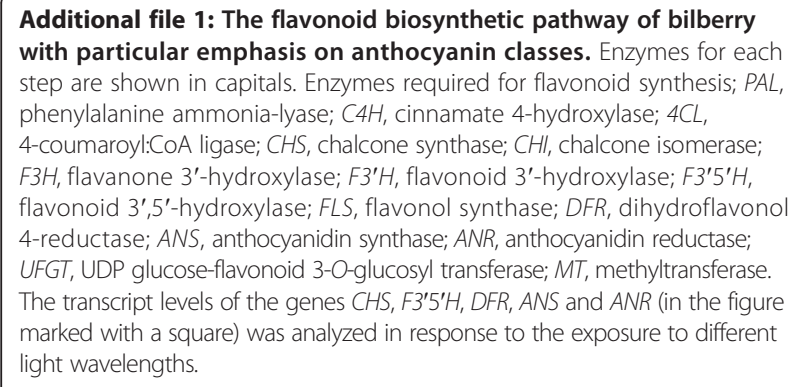

Additional file 2: Relative transcript abundance of the flavonoid pathway genes $V m C H S, V m F 3^{\prime} 5^{\prime} H, V m D F R, V m A N S$ and $V m A N R$, and the transcription factor $V m M Y B 2$ in bilberry fruits (at stage 2) after 6, 12, 24 and $48 \mathrm{~h}$ under different light conditions. Data represent average and SD values of samples collected from two locations (see Methods).

Additional file 3: UPLC-MS/MS data for anthocyanin quantification. In case of two MRM transitions for a given compound, the first was used as quantifier and the second as qualifier. RT = retention time, $C V=$ cone voltage, $\mathrm{CE}=$ collision energy, $\mathrm{Std}=$ standard curve.

Additional file 4: Sequences of the primers used in qPCR to determine gene transcripts.

\section{Abbreviations}

UPLC-MS/MS: Ultra performance liquid chromatography - tandem mass spectrometer; Dp: Delphinidin; Cy: Cyanidin; Pt: Petunidin; Pn: Peonidin; Mv: Malvidin; Pg: Pelargonidin; Glu: Glucose; Gal: Galactose; Ara: Arabinose; Coum: Coumaroyl; DW: Dry weight; VmCHS: Vaccinium myrtillus chalcone synthase; VmF3'5'H: Vaccinium myrtillus flavonoid 3'5'-hydroxylase; VmDFR: Vaccinium myrtillus dihydroflavonol 4-reductase; VmANS: Vaccinium myrtillus anthocyanidin synthase; VmANR: Vaccinium myrtillus anthocyanidin reductase; VmMYB2: Vaccinium myrtillus MYB2 transcription factor; VmACT: Vaccinium myrtillus actin; MRM: Multiple reaction monitoring.

\section{Competing interests}

The authors declare that they have no competing interests.

\section{Authors' contributions}

LZ performed most of the experimental work together with interpretation of data, was involved in the design of the work, and most of writing and editing; MS contributed in performing the experiment and gene expression analyses; EC gave support with the metabolic analyses and contribution to the interpretation of the data; KK, SM and LG gave contribution with the interpretation of the data; $\mathrm{L}$ and $\mathrm{HH}$ provided contribution to the conception and the design of the work. All authors attended to the writing of the manuscript and read and approved the final manuscript.

\section{Acknowledgements}

Our special thanks to Matti Rauman, for his professionalism and for the great contribution to this project by setting up the light systems. Kone Foundation (to $L$ ), the Finnish Doctoral Program in Plant Biology and the STSM (Short Term Scientific Program) program within the COST action FA1006 (PlantEngine) (to LZ) are acknowledged for the financial support.

\section{Author details}

${ }^{1}$ Department of Biology, University of Oulu, PO Box 3000, FI-90014 Oulu, Finland. 'Plant Molecular Science, Centre for Systems and Synthetic Biology, Royal Holloway University of London, TW20 OEX Egham, UK. ${ }^{3}$ Fondazione Edmund Mach, Research and Innovation Center, via E. Mach 1, 38010 S Michele all'Adige, TN, Italy. ${ }^{4}$ Climate laboratory, Department of Arctic and Marine Biology, UiT the Arctic University of Norway, NO-9037 Tromsø, Norway. ${ }^{5}$ Norwegian Institute for Agricultural and Environmental Research, Bioforsk Nord Holt, Box 2284, NO-9269 Tromsø, Norway.

Received: 31 October 2014 Accepted: 10 December 2014 Published: 16 December 2014

\section{References}

1. Steyn WJ: Prevalence and functions of anthocyanins in fruits. In Anthocyanins: Biosynthesis, Functions, and Applications. Edited by Winefield C, Davies K, Gould K. New York: Springer; 2009:85-105.

2. Dai J, Mumper RJ: Plant phenolics: extraction, analysis and their antioxidant and anticancer properties. Molecules 2010, 15:7313-7352.

3. de Pascual-Teresa S, Moreno DA, Garcia-Viguera C: Flavanols and anthocyanins in cardiovascular health: a review of current evidence. Int J Mol Sci 2010, 11:1679-1703.

4. Moze S, Polak T, Gasperlin L, Koron D, Vanzo A, Poklar Ulrih N, Abram V: Phenolics in Slovenian bilberries (Vaccinium myrtillus L.) and blueberries (Vaccinium corymbosum L.). J Agric Food Chem 2011, 59:6998-7004.

5. Skrede G, Martinsen BK, Wold AB, Birkeland SE, Aaby K: Variation in quality parameters between and within 14 Nordic tree fruit and berry species. Acta Agric Scand B 2012, 62:193-208.

6. Jaakola L, Määttä K, Pirttilä AM, Törrönen R, Kärenlampi S, Hohtola A: Expression of genes involved in anthocyanin biosynthesis in relation to anthocyanin, proanthocyanidin, and flavonol levels during bilberry fruit development. Plant Physiol 2002, 130:729-739.

7. Jaakola L, Poole M, Jones MO, Kämäräinen-Karppinen T, Koskimäki JJ, Hohtola A, Häggman H, Fraser PD, Manning K, King GJ, Thomson H, Seymour GB: A SQUAMOSA MADS Box gene involved in the regulation of anthocyanin accumulation in bilberry fruits. Plant Physiol 2010 153:1619-1629.

8. Lätti AK, Riihinen KR, Kainulainen PS: Analysis of anthocyanin variation in wild populations of bilberry (Vaccinium myrtillus L.) in Finland. J Agric Food Chem 2008, 56:190-196.

9. Åkerström A, Jaakola L, Bång U, Jaderlund A: Effects of latitude-related factors and geographical origin on anthocyanidin concentrations in fruits of Vaccinium myrtillus L. (bilberries). J Agric Food Chem 2010, 58:11939-11945.

10. Uleberg E, Rohloff J, Jaakola L, Trost K, Junttila O, Häggman H, Martinussen I: Effects of temperature and photoperiod on yield and chemical composition of northern and southern clones of bilberry (Vaccinium myrtillus L.). J Agric Food Chem 2012, 60:10406-10414.

11. Davies KM, Schwinn KE: Transcriptional regulation of secondary metabolism. Funct Plant Biol 2003, 30:913-925.

12. Koes R, Verweij W, Quattrocchio F: Flavonoids: a colorful model for the regulation and evolution of biochemical pathways. Trends Plant Sci 2005 10:236-242.

13. Lillo C, Lea US, Ruoff P: Nutrient depletion as a key factor for manipulating gene expression and product formation in different branches of the flavonoid pathway. Plant Cell Env 2008, 31:587-601.

14. Jaakola L, Hohtola A: Effect of latitude on flavonoid biosynthesis in plants. Plant Cell Env 2010, 33:1239-1247.

15. Martz F, Jaakola L, Julkunen-Tiitto R, Stark S: Phenolic composition and antioxidant capacity of bilberry (Vaccinium myrtillus) leaves in northern europe following foliar development and along environmental gradients. J Chem Ecol 2010, 36:1017-1028.

16. Zoratti L, Karppinen K, Luengo Escobar A, Häggman H, Jaakola L: Light-controlled flavonoid biosynthesis in fruits. Front Plant Sci 2014, 5:1-16.

17. Jiao Y, Lau OS, Deng XW: Light-regulated transcriptional networks in higher plants. Nat Rev Gens 2007, 8:217-230.

18. Hong GJ, Hu WL, Li JX, Chen XY, Wang LJ: Increased accumulation of artemisinin and anthocyanins in artemisia annua expressing the arabidopsis blue light receptor CRY1. Plant Molec Biol Rep 2009, 27:334-341. 
19. Cominelli E, Gusmaroli G, Allegra D, Galbiati M, Wade HK, Jenkins Gl, Tonelli C: Expression analysis of anthocyanin regulatory genes in response to different light qualities in Arabidopsis thaliana. J Plant Physiol 2008, 165:886-894.

20. Zhou Y, Singh BR: Red light stimulates flowering and anthocyanin biosynthesis in American cranberry. Plant Growth Regul 2002, 38:165-171.

21. Meng XC, Xing T, Wang XJ: The role of light in the regulation of anthocyanin accumulation in Gerbera hybrida. Plant Growth Regul 2004, 44:243-250

22. Koyama K, Ikeda H, Poudel PR, Goto-Yamamoto N: Light quality affects flavonoid biosynthesis in young berries of Cabernet Sauvignon grape. Phytochemistry 2012, 78:54-64.

23. Kondo S, Tomiyama H, Rodyoung A, Okawa K, Ohara H, Sugaya S, Terahara $\mathrm{N}$, Hirai N: Abscisic acid metabolism and anthocyanin synthesis in grape skin are affected by light emitting diode (LED) irradiation at night. J Plant Physiol 2014, 171:823-829.

24. Li Q, Kubota C: Effects of supplemental light quality on growth and phytochemicals of baby leaf lettuce. Env Exp Bot 2009, 67:59-64.

25. Kadomura-Ishikawa Y, Miyawaki K, Noji S, Takahashi A: Phototropin 2 is involved in blue light-induced anthocyanin accumulation in Fragaria $\mathrm{x}$ ananassa fruits. J Plant Res 2013, 126:847-857.

26. Zhou B, Li Y, Xu Z, Yan H, Homma S, Kawabata S: Ultraviolet A-specific induction of anthocyanin blosynthesis in the swollen hypocotyls of turnip (Brassica rapa). J Exp Bot 2007, 58:1771-1781.

27. Jaakola L, Määttä-Riihinen K, Kärenlampi S, Hohtola A: Activation of flavonoid biosynthesis by solar radiation in bilberry (Vaccinium myrtillus L.) leaves. Planta 2004, 218:721-728.

28. Vrhovsek U, Masuero D, Gasperotti M, Franceschi P, Caputi L, Viola R, Mattivi F: A versatile targeted metabolomics method for the rapid quantification of multiple classes of phenolics in fruits and beverages. J Agric Food Chem 2012, 60:8831-8840.

29. Mikulic-Petkovsek M, Slatnar A, Stampar F, Veberic R: HPLC-MSn identification and quantification of flavonol glycosides in 28 wild and cultivated berry species. Food Chem 2012, 135:2138-2146.

30. Arapitsas P, Perenzoni D, Nicolini G, Mattivi F: Study of sangiovese wines pigment profile by UHPLC-MS/MS. J Agric Food Chem 2012, 60:10461-10471.

31. Hokkanen J, Mattila S, Jaakola L, Pirttilä AM, Tolonen A: Identification of phenolic compounds from lingonberry (Vaccinium vitis-idaea L.), bilberry (Vaccinium myrtillus L.) and hybrid bilberry (Vaccinium $\mathrm{x}$ intermedium Ruthe L.) leaves. J Agric Food Chem 2009, 57:9437-9447.

32. Du QJG, Winterhalter P: Isolation of two anthocyanin sambubiosides from bilberry (Vaccinium myrtillus) by high-speed counter-current chromatography. J Chromatogr A 2004, 1045:59-63.

33. Bada JC, Leon-Camacho M, Copovi P, Alonso L: Characterization of berry and currant seed oils from asturias, spain. Int J Food Prop 2014, 17:77-85.

34. Thain SC, Murtas G, Lynn JR, McGrath RB, Millar AJ: The circadian clock that controls gene expression in Arabidopsis is tissue specific. Plant Physiol 2002, 130:102-110

35. Taulavuori K, Sarala M, Taulavuori E: Growth responses of trees to arctic light environment. Progr Bot 71 2010, 71:157-168.

36. Alberts T, Raspé O, Jacquemart AL: Clonal diversity and genetic structure in Vaccinium myrtillus populations from different habitats. Belgian $J$ Botany 2004, 137:155-162.

37. Tian QG, Giusti MM, Stoner GD, Schwartz SJ: Screening for anthocyanins using high-performance liquid chromatography coupled to electrospray ionization tandem mass spectrometry with precursor-ion analysis, product-ion analysis, common-neutral-loss analysis, and selected reaction monitoring. J Chromatogr A 2005, 1091:72-82.

38. Jaakola L, Pirttilä AM, Halonen M, Hohtola A: Isolation of high quality RNA from bilberry (Vaccinium myrtillus L.) fruit. Mol Biotech 2001, 19:201-203.

doi:10.1186/s12870-014-0377-1

Cite this article as: Zoratti et al:: Monochromatic light increases anthocyanin content during fruit development in bilberry. BMC Plant Biology 2014 14:377.

\section{Submit your next manuscript to BioMed Central and take full advantage of:}

- Convenient online submission

- Thorough peer review

- No space constraints or color figure charges

- Immediate publication on acceptance

- Inclusion in PubMed, CAS, Scopus and Google Scholar

- Research which is freely available for redistribution

Submit your manuscript at www.biomedcentral.com/submit
C BioMed Central 